\section{Wie handeln?}

Kooperationsprobleme zwischen Kinder- und Jugendhilfe und
Kinder- und Jugendpsychiatrie/Psychotherapie

Als Vertragsstaat der Kinderrechtskonvention der Vereinten Nationen erkennt Deutschland das Recht des Kindes auf das erreichbare Höchstma $\beta$ an Gesundheit an (Artikel 24 Absatz 1). Das bedeutet, dass überall dort, wo staatliche Stellen unmittelbar Verantwortung für Kinder und Jugendliche tragen, diese für ein förderliches Umfeld der ihnen anvertrauten Kinder zuständig sind. Zu den Verantwortungsträgern gehören neben der Behindertenhilfe (gesetzlich flankiert durch das SGB IX), die Kinder- und Jugendhilfe (KJH) (durch das SGB III) sowie die Kinder- und Jugendpsychiatrie und -Psychotherapie (KJPP) (durch das SGB V).

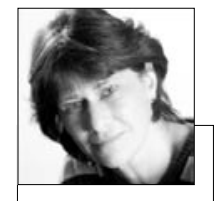

Margret Dörr *1956

Dr., Professorin an der schule für Soziale Arbeit in Mainz. Arbeitsschwerpunkte: Theorien Sozialer Arbeit; Biographierie, Psychoanalytische (Sozial-) Pädagogik. margret.doerr @t-online.de Katholischen Fachhochund Sozialisationstheo-
In geteilter Verantwortung haben die Akteure der jeweiligen Arbeitsfelder den Auftrag, auch denjenigen Kindern und Jugendlichen, die unter schwierigen Lebensbedingungen aufwachsen müssen und diversen Gefahren ausgesetzt waren und sind, einen ganz besonderen Unterstützungsbedarf anzubieten. Hierbei sind sie verpflichtet, als Garanten für die Kinder/Jugendlichen und ihre Grundrechte aufzutreten. Aber biographische Studien z.B. von Heimkindern (vgl. u.a. wolf 2007) machen auf den skandalösen Sachverhalt aufmerksam, dass vor allem psychosozial hoch belastete und potentiell traumatisierte Heranwachsende als „Problemfälle“ - in die für sie unheilvollen Mühlen eines „Verschiebebahnhofs“ geraten. Dieser konstituiert sich an den Übergängen, zwischen den Helfersystemen meist dann, wenn professionelle Ak,Kinderschutzes', an ihre Grenzen gerazur Abwehr von Kindeswohlgefährdung oder zur Eröffnung von Entwicklungsund Bildungschancen oder Behandlung gedacht waren, können (strukturelle) Eigendynamiken entwickeln, die für die teure mit ihren jeweiligen Konzepten des ten: Staatliche Eingriffe, die „eigentlich“
Betroffenen ein großes Potential an (Re-) Viktimisierungsrisiken enthalten.

So hat die geteilte Verantwortung nicht selten Versorgungseinbrüche an den Übergängen zur Folge. Statt notwendiger Unterstützungsmaßnahmen durch Kooperation werden sie den unproduktiven Ansprüchen disziplinärer und professioneller Vorherrschaft der miteinander konkurrierenden Hilfesysteme ausgesetzt. „Hier arbeiten offensichtlich zwei Systeme teilweise aneinander vorbei, bedienen sich multipler ideologischer Schuldzuweisungen und benutzen den jeweils anderen als Lückenbüßer an der Grenze der eigenen Ressourcen.“ (FEGERT 2007: 7)

Gerade dieser (Eigen-)Anteil der professionellen Praxen bei der Konstitution eines ,schwierigen' Falls - sei es eines „Falles der stationären Jugendhilfe“ oder eines „Falles der Kinder- und Jugendpsychiatrie“ - wird häufig verleugnet und meist als stigmatisierende Attribuierung in die betroffenen Kinder und Jugendlichen („Erziehungsresistente“) hineinverlegt.
Ein Sachverhalt, der aus der Perspektive der Betroffenen unschwer als ,unterlassene Hilfeleistung“ oder als „Kindeswohlgefährdung“ begriffen werden muss.

Welche Anforderungen an beide Sektoren $\mathrm{zu}$ formulieren sind, um Schnittstellen nicht zu Bruchstellen für die Betroffenen werden zu lassen, aber auch welche Fallstricke die jeweiligen Akteure zu meistern haben, um eine gesicherte Kooperation im Hinblick auf entwicklungs- und gesundheitsförderliche Bedingungen auch für "schwierige“ Kinder und Jugendliche zu verankern, wird in den Beiträgen beispielhaft und aus unterschiedlichen Perspektiven in den Blick genommen. Während Sabine Ader und Martin Klein von den wechselseitigen „Erwartungs-Enttäuschungen“ der Akteure der KJH und KJPP ausgehen, und darüber sehr anschaulich bestehende Dilemmata offenlegen sowie notwendige Anstrengungen der Kooperation aufzeigen, um „organisierte Verantwortungslosigkeit“ aufzubrechen, werfen Ulrike Becker und Uwe Sander ihren Blick auf die zerstörerische Odyssee durch vielfältige Hilfemaßnahmen besonders ,problematischer" Jugendliche. Dabei thematisieren sie sowohl die oft lähmende und Resignation erzeugende Hilflosigkeit der professionellen Akteure als auch die Chancen kreativer Neuorientierung in der gemeinsamen Praxis mit diesen Jugendlichen. Wie sehr eine Kooperation zwischen Jugendhilfe und Jugendpsychiatrie des Wissens um theoretische Sichtweisen sowie Arbeitbedingungen bedarf, um eine konstruktive Zusammenarbeit zu gestalten, zeigt Martina Steger am Beispiel der Bedeutung fachärztlicher Stellungnahmen zur Planung von Eingliederungshilfen nach $\S 35$ a SGB VIII. ส้ ส

\section{Literatur}

FEGERT, J.M. (2007).

Vorwort zu Marc Schmid: Psychische Gesundheit von Heimkindern

Eine Studie zur Prävalenz psychischer Störungen in der stationären Jugendhilfe (S. 7-11). Weinheim, München

WOLF, K. (2007).

Metaanalyse von Fallstudien erzieherischer Hilfen hinsichtlich von Wirkungen und „wirkmächtigen“ Faktoren aus Nutzersicht. Münster 